\title{
System Test of a Prototype LHCb RICH Detector
}

\author{
Mitesh Patel, CERN, Geneva, Switzerland *
}

\begin{abstract}
A prototype of the LHCb Ring Imaging Cherenkov detector has been constructed. The prototype module contained a pre-production Pixel Hybrid Photon Detector, mounted on the final photon detector mechanics. The photon detector was read out at the full LHC speed of $40 \mathrm{MHz}$ using the full prototype on-detector RICH electronics readout chain. The readout uses radiation-tolerant FPGA technology, 1.6GHz optical links and $40 \mathrm{MHz}$ trigger-timing and control (TTC). The photon detector was mounted in a gas vessel and Cherenkov rings have been observed from an $\mathrm{N}_{2}$ radiator using electron and pion beams.
\end{abstract}

Index Terms - Ring Imaging Cherenkov; Pixel Hybrid Photon Detectors.

\section{INTRODUCTION}

$\mathbf{T}$ HE LHCb experiment [1] will make high precision studies of $\mathrm{CP}$ violation and other rare phenomena in $\mathrm{B}$ meson decays. Particle identification, in particular, $\pi-K$ separation capability, in the momentum range from a few to $\sim 100 \mathrm{GeV} / \mathrm{c}$, is essential for this physics programme. In order to provide this capability, two Ring Imaging CHerenkov ( $\mathrm{RICH}$ ) detectors will be employed [2]. Three different radiator materials: silica aerogel, and the fluorocarbon gases $\mathrm{C}_{4} \mathrm{~F}_{10}$ and $\mathrm{CF}_{4}$, will be used to produce Cherenkov light from charged particles traversing the detectors. The resulting single photons must be detected for wavelengths in the range 200 to $600 \mathrm{~nm}$, over a total area of $2.8 \mathrm{~m}^{2}$. The photon detectors distributed over this area are required to have a granularity of $2.5 \mathrm{~mm} \times 2.5 \mathrm{~mm}$, a time resolution better than the LHC bunch crossing rate of $25 \mathrm{~ns}$ and an active-to-total area ratio of $\sim 70 \%$. In addition, the on-detector electronics must be radiation tolerant to a dose of $\sim 3 \mathrm{kRad} /$ year.

Photon detector prototypes that satisfy these constraints have been developed and subjected to extensive laboratory and beam tests. A transistion must now be made from these prototypes to an integrated system in which $\sim 550$ detectors are mounted and maintained in position, are read out with the full on-detector electronics chain, and are thermally and electrically stable.

A prototype $\mathrm{RICH}$ detector, employing pre-production photon detectors with their associated mechanics, cooling and insulation, together with the prototype on-detector readout electronics, has been constructed to test these integration issues. This paper reports the first of two phases of test beam operation of this prototype. In the following sections the photon detectors and then the prototype RICH detector are described together with solutions to some of the integration issues. The results from the first phase of test beam operation are then presented and the programme for the second phase is also described.

\footnotetext{
* on behalf of the LHCb RICH group
}

\section{The Hybrid Photon Detector}

The photon detector solution adopted for the $\mathrm{LHCb} \mathrm{RICH}$ detectors, is the novel Pixel Hybrid Photon Detector (HPD) which has been developed in close collaboration with industry. The HPD consists of a pixelated silicon detector anode assembly which is encapsulated in a vacuum envelope (figure 1). The silicon sensor chip of the anode assembly is divided into $256 \times 32$ pixels of $62.5 \times 500 \mu \mathrm{m}^{2}$, with each pixel bump-bonded to one channel of the LHCbPIX1 binary readout chip [4]. Groups of eight pixels are OR-ed together in this chip, effectively creating 1024 pixels of $500 \times 500 \mu \mathrm{m}^{2}$.

The HPD tube has a $7 \mathrm{~mm}$-thick spherical quartz entrance window with an S20 multialkali photocathode deposited on the inside. The active diameter of this window is $75 \mathrm{~mm}$ giving an intrinsic active area of the HPD of $>80 \%$. In a hexagonal close packing arrangement the tube active-to-total surface ratio is then 0.7 . The typical quantum efficiency is $\sim 25 \%$ at $270 \mathrm{~nm}$. Photoelectrons emitted from the photocathode are accelerated onto the anode assembly by a $20 \mathrm{kV}$ cross-focussing electron optics system. This produces $\sim 5000 \mathrm{e}^{-}$in the silicon. The resulting image on the anode has a demagnification factor $\sim 5$ with distortions at the $<10 \%$ level which can be corrected for off-line.

A number of HPD prototypes have been subject to a programme of laboratory and beam tests which have all indicated that the device complies with LHCb's requirements [3]. The detection efficiency after photon conversion has been determined to be $88 \%$. Aging tests simulating ten years of $\mathrm{LHCb}$ operation showing no observable deterioration in this efficiency.

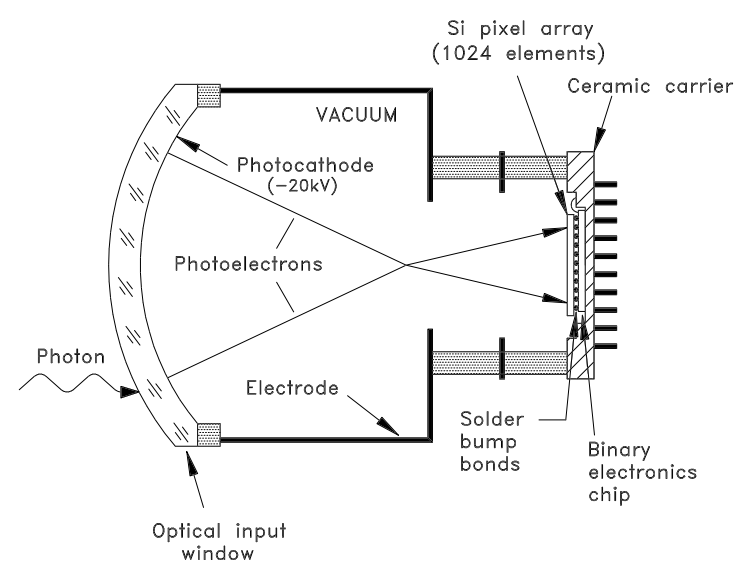

Fig. 1. Schematic of the Pixel HPD, illustrating photoelectron trajectories. 


\section{The PRototype RICH Detector}

Beam tests were performed at the CERN-PS in the T9 facility which provided $10 \mathrm{GeV} / \mathrm{c}$ negative pions and electrons. The Cherenkov detector used in the test beam is shown schematically in figure 2 .

Although the prototype was initially employed in beam tests, its size was such that it could be used as a testbed for integration issues in the absence of beam particles. The detector housing was therefore large enough to allow a full complement of HPDs to be mounted in the full-sized assembly that will be used in the LHCb detector. This final mechanics scheme, referred to as a column, is described in section III-A.

The light-tight vessel used to contain the Cherenkov radiator was constructed from an aluminum frame covered in aluminum sheets. The beam particles entered the vessel through a thin aluminum foil and impinged on the mirror centre at an angle of $13.4^{\circ}$ to the mirror axis. Initial alignment relative to the particle beam axis was performed using a laser beam.

The parabolic mirror used had a focal length $\mathrm{f}=1016 \mathrm{~mm}$ and a diameter of $200 \mathrm{~mm}$. The mirrors in the final detector will be spherical, however, the coating was the one that which will eventually be used. The reflectivity of the mirror was measured to be more than $90 \%$ in the range $225-450 \mathrm{~nm}$. The detector plane was fixed at a distance of $1047 \mathrm{~mm}$ from the mirror centre with the dimensions chosen to ensure that saturated electron rings could be fully contained within a single HPD with an $\mathrm{N}_{2}$ gas radiator.

The radiator volume was sealed with a $5 \mathrm{~mm}$ thick quartz plate through which the Cherenkov photons entered the detector housing. The Cherenkov radiator used in the first phase of

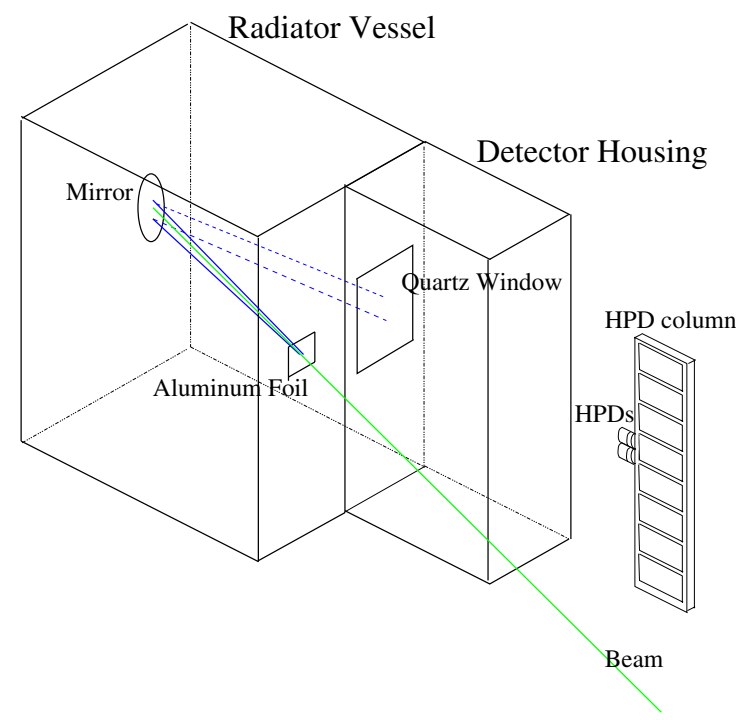

Fig. 2. Schematic of the prototype RICH detector indicating the beam and passage of Cherenkov light. The aluminum foil entrance window and quartz window separating the radiator vessel and dectector housing are indicated. A single column assembly is also shown with two HPDs mounted. This assembly slides into the dectector housing on rails and, in position, the HPD photocathode is $50 \mathrm{~mm}$ from the quartz window. test beam operation reported here was $\mathrm{N}_{2}$ gas. In the second phase $\mathrm{C}_{4} \mathrm{~F}_{10}$ will be used, as in the final upstream LHCb RICH detector. Electron rings will then be spread over four HPDs.

\section{A. Mechanics}

Pairs of HPDs are mounted on an aluminum supporting frame which can house a total of eight sub-assembly pairs arranged in columns (figure 3). These sub-assemblies are mechanically fixed to the HPDs back-plate through aluminum spacers (see figure 4). The frame also houses one readout electronics board (Level-0 board - see section III-B) which serves two HPDs, as well as the associated high and low-voltage power distribution boards.

The prototype detector contained three sets of rails for these supporting frames. All HPD cables were routed out from the HPD through its back-plate and then by the side of the supporting frame. The columns could therefore each be individually pulled backward without disturbing neighbouring columns. For the first phase of the test beam reported here only one HPD was mounted in the prototype RICH detector on a single column. The second phase will make use of $\sim 10$ pre-production HPDs in a hexagonal close packing arrangement with a tube pitch of $89.5 \mathrm{~mm}$. This is identical to that which will be used in the final RICH detectors. The mechanical tolerance of all subcomponents was of order $0.1 \mathrm{~mm}$. The columns used in the beam tests were the first of the final design to be fabricated. The test period therefore allowed the first tests to be made of the HPD mounting scheme. No mechanical conflicts were found.

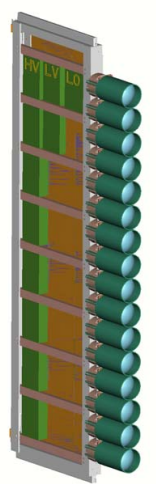

Fig. 3. The HPD column assembly. A fully occupied column, with 16 HPDs is shown.

\section{B. The Readout Electronics}

The first stage of the $\mathrm{RICH}$ readout system is the binary front-end pixel chip, LHCbPIX1, which is encapsulated inside the Pixel HPD vacuum envelope. The readout chip discriminates single photoelectron hits and tags them with the triggered LHC bunch crossing identifier. The chip has a front-end amplifier with shaping time $25 \mathrm{~ns}$, and a discriminator threshold of $\sim 1500 \mathrm{e}^{-}$with a pixel-to-pixel spread of $200 \mathrm{e}^{-}$. This allows detection of single photoelectrons, including those that 


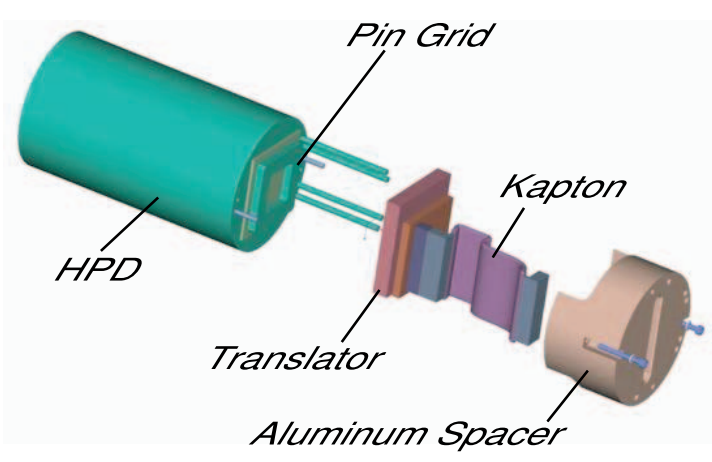

Fig. 4. The HPD mounting scheme.

experience charge sharing among neighbouring pixels (which typically have lower signal amplitudes) and those that backscatter, with a good signal-to-noise ratio. The pixel chip is fabricated in $0.25 \mu \mathrm{m}$ CMOS technology using a layout adapted for tolerance to ionising radiation and single event upset.

The test beam readout scheme is shown in figure 5. A pair of HPDs are connected via a translator module to the Level-0 readout board, mounted on-detector. The translator module is passive, and connects the HPD to the Level-0 board via a pingrid array on the HPD and $\sim 10 \mathrm{~cm}$ of kapton cable (figure 4).

The Level-0 board interfaces the HPD pixel chips to the trigger, timing and control and the experimental control systems, and receives and distributes the local low-voltage power to the binary chip and controls it via the JTAG protocol [5].

The Level-0 functionality is implemented on the so-called PINT chip, a radiation-tolerant ACTEL fuse-link Field Programmable Gate Array (FPGA). The Level-0 board further multiplexes the data onto optical links to the off-detector (Level-1) electronics.

The synchronisation of the front-end electronics and the local distribution of the clocks and triggers is achieved using the Timing, Trigger and Control Receiver ASIC (TTCrx) [6]. On a Level-0 trigger accept, the data from a pair of pixel chips are multiplexed out at a $40 \mathrm{MHz}$ rate as two 32-bit streams. The multiplex grouping of 32 is chosen to match the maximum average LHCb Level-0 trigger rate of $1 \mathrm{MHz}$. The GigabitEthernet protocol is used to drive the optical fibres at a $1.6 \mathrm{GHz}$ rate. The time available for reading out the data from the Level0 into the Level- 1 is $900 \mathrm{~ns}$, determined by the average Level- 0 accept rate in the LHCb experiment.

The Level-1 readout board receives multiplexed data from the Level-0 via the optical links, buffers and processes the data, and then transports the data to the LHCb DAQ system. For the purposes of these tests, only the Level-1 optical receiver was used. The receiver card was mounted onto an S-link to PCI bridge (FLIC) [7]. Triggered Level-0 events were transferred to the FLIC as soon as they were received and the data were buffered there until the end of the PS spill. They were then copied into the local memory of a readout PC through the PCI interface and then copied to disk.

During test beam operation, data were driven through the

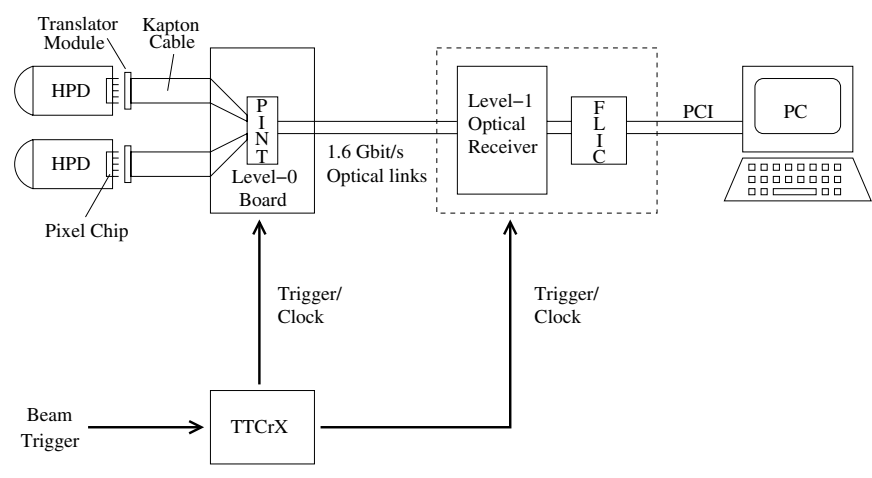

Fig. 5. Schematic of the RICH readout electronics.

readout system at full LHCb speed with the pixel chip running at $40 \mathrm{MHz}$ and the optical links channelling the data to the receiver at the full $1.6 \mathrm{GHz}$ rate. However, additional hits were observed in neighbouring pixels for some of the channels. While investigation of the origin of this problem is currently on going, preliminary indications are that a faulty distribution of a GTL reference is responsible for this effect. This will be corrected for the next iteration of the Level-0 board.

\section{Cooling}

The 16 HPDs in a fully equipped LHCb RICH detector column will output $150 \mathrm{~W}$. Each HPD column will therefore be fitted with a cooling plate as shown in figure 6 . These will also serve as electromagnetic radiation shields.

The plates are formed from two copper skins with an aluminum honeycomb in between and have a total thickness of $6 \mathrm{~mm}$. The two parallel copper tubes that run through the honeycomb will be supplied with $\mathrm{C}_{6} \mathrm{~F}_{14}$. In the final detectors a thermal mat will be used to provide thermal contact between components on the on-detector electronics and the copper skin of the cooling plates.

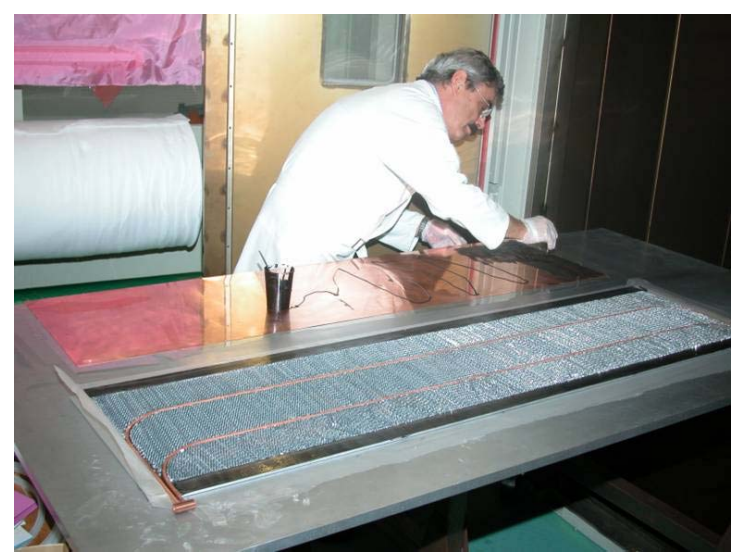

Fig. 6. A cooling plate during its construction. A thermally conductive adhesive is being applied to the outer copper skin which sits on top of the aluminum honeycomb that can be seen around the copper pipes. The inlet for the cooling pipe is visible in the lower left corner. 


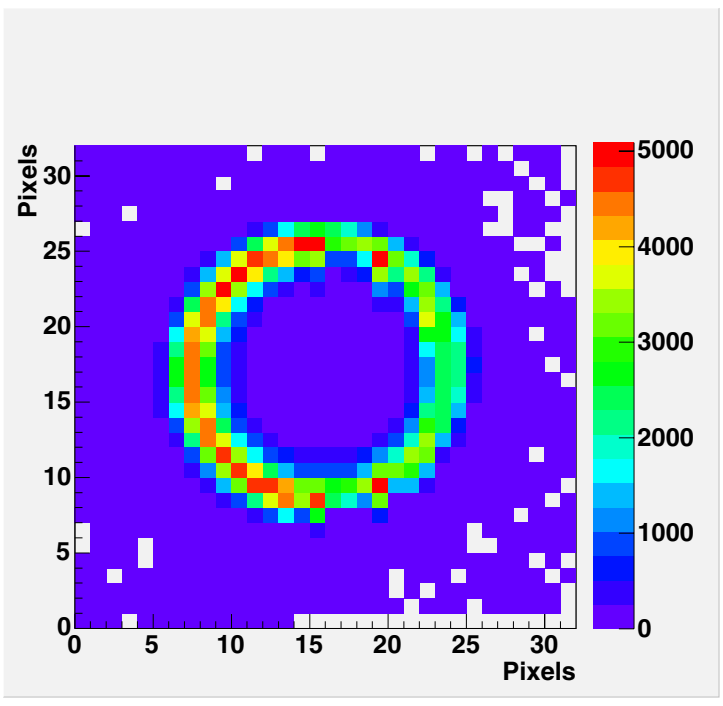

Fig. 7. The Cherenkov ring observed on the pixel chip from an electron run, superimposing 25000 events.

\section{ObServation of Cherenkov RingS}

The Cherenkov rings observed on the pixel detector from an electron run containing 25000 triggers is shown in figure 7 . The readout problem described gives rise to many more hits on the left hand side of the pixel chip.

Correcting for the readout problem offline the ring shown in figure 8 is recovered. The ring from a pion run, similarly corrected, is shown in figure 9. The difference in the number of hits at the top and bottom of these rings compared to the sides results from the imperfect focussing in the horizontal direction. This is created by the tilt of the mirror with respect to the

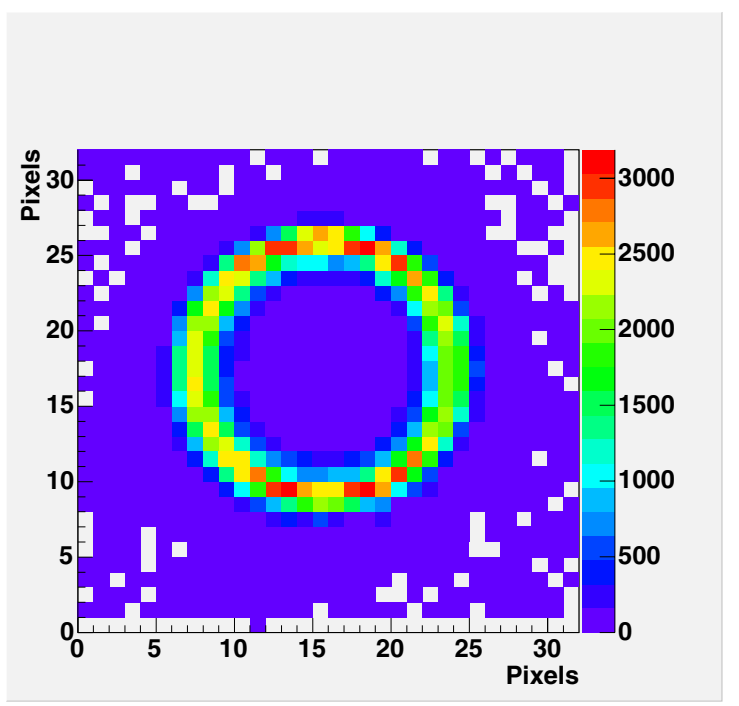

Fig. 8. The Cherenkov ring observed on the pixel chip from an electron run corrected for the readout problem.
TABLE I

\begin{tabular}{c||c|c}
\hline \multicolumn{1}{c||}{} & \multicolumn{2}{c}{ Ring Radius/mm } \\
Particle & Observed & Expected \\
\hline \hline Electron & $4.0 \pm 0.6$ & $4.2 \pm 0.1$ \\
\hline Pion & $3.3 \pm 0.5$ & $3.5 \pm 0.1$ \\
\hline
\end{tabular}

TABLE II

\begin{tabular}{c||c|c}
\hline \multicolumn{1}{c||}{} & \multicolumn{2}{c}{ Number of Photoelectrons } \\
Particle & Observed & Expected \\
\hline \hline Electron & $12.6 \pm 0.1$ & $12.1 \pm 1.1$ \\
\hline Pion & $9.1 \pm 0.1$ & $7.9 \pm 0.8$ \\
\hline
\end{tabular}

detector plane.

Preliminary estimates of the ring radii observed and those expected are given in Table I. The data are in good agreement. The corresponding Cherenkov angles are 23.9 and $19.4 \mathrm{mrad}$ for the electron and pion rings respectively. The average number of photoelectrons observed and expected for both electron and pion runs are given in Table II. The data are again in good agreement. Detailed analysis of the data is underway in order to refine these measurements and to determine the detection efficiency.

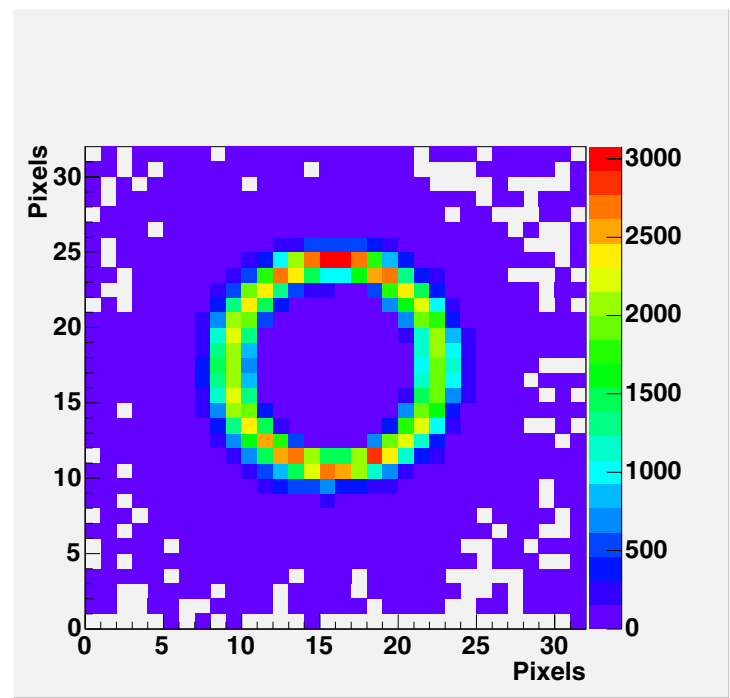

Fig. 9. The Cherenkov ring observed on the pixel chip from a pion run corrected for the readout problem. 


\section{CONCLUSiON}

A test of a prototype $\mathrm{LHCb} \mathrm{RICH}$ detector, employing a single pre-production photon detector with its associated mechanics and readout electronics, has been made. Using $10 \mathrm{GeV} / \mathrm{c}$ electron and pion beams from the CERN-PS T9 facility, the photon detector performance has been observed to be similar to that seen in the laboratory. The photon detector was mounted on a prototype mechanical frame which can hold 16 photon detectors, in pairs. The data were read out at the full LHC speed of $40 \mathrm{MHz}$ with a prototype on-detector readout system, using $1.6 \mathrm{GHz}$ optical fibres and a receiver to extract the data for storage. Cherenkov rings have been observed with little background and ring radii and photoelectron yields in line with expectations.

A second phase of beam tests will be used to read out ten photon detectors using a $\mathrm{C}_{4} \mathrm{~F}_{10}$ radiator. With this larger number of units, tests of the close packing of multiple photon detectors in three columns will be possible, as well as tests of the cooling and high voltage insulation schemes.

\section{ACKNOWLEDGMENT}

The LHCb RICH Group would like to thank Andre Braem, the CERN-PS division for their provision of the test beam and the $\mathrm{LHCb}$ online team.

\section{REFERENCES}

[1] S.Amato et al., LHCb technical proposal, CERN-LHCC-98-4, CERN, 1998

[2] The LHCb Collaboration, LHCb technical design report: Reoptimized detector design and performance, CERN-LHCC-2003-030, 2003

[3] M.Moritz et al., Performance Study of New Pixel Hybrid Photon Detector Prototypes for the LHCb RICH Counters, Nucl.Instrum.Meth.A442,164170,2000

[4] K.Wyllie et al., Readout of the LHCb Pixel Hybrid Photon Detectors International Workshop on Radiation Imaging Detectors, Conference Proceedings, to be published in Nucl.Instrum.Meth.A

[5] "JTAG: IEEE Standard Test Access Port and Boundary Scan Architecture", IEEE Std., 1149.1,1990.

[6] J.Christiansen, et al., "TTCrx Reference Manual", RD12 project. http://www.cern.ch/TTC/TTCrx_manual3.0.pdf

[7] A.Guirao et al.,Readout of high speed S-LINK data via a buffered PCI card, Conference Proceedings, Frascati, PCaPAC 2002 